Review Article

\title{
Four-Rooted Maxillary First Molars: A Systematic Review and Meta-Analysis
}

\author{
Gabriel Magnucki $\mathbb{D}^{1}$ and Sven V. K. Mietling $\mathbb{D}^{2}$ \\ ${ }^{1}$ Zahnzentrum Schomaker and Magnucki, Bahnhofstr. 16-18, Bassum 27211, Germany \\ ${ }^{2}$ Zahnarztpraxis Mietling, Hauptstr. 117, Hille-Oberlübbe 32479, Germany \\ Correspondence should be addressed to Gabriel Magnucki; gabriel_magnucki@gmx.de and Sven V. K. Mietling; s.mietling@ \\ gmx.de
}

Received 28 April 2020; Revised 8 November 2020; Accepted 17 December 2020; Published 21 January 2021

Academic Editor: Sreekanth Kumar Mallineni

Copyright ( $) 2021$ Gabriel Magnucki and Sven V. K. Mietling. This is an open access article distributed under the Creative Commons Attribution License, which permits unrestricted use, distribution, and reproduction in any medium, provided the original work is properly cited.

\begin{abstract}
Objectives. The majority of human maxillary first molars is usually described as having three roots, but different morphologies were documented in several studies and case reports. One very rare and less investigated anatomical anomaly is the occurrence of four radicular structures in the upper first molars. This communication aimed to define the prevalence of four-rooted maxillary first molars on a meta-analytical basis. The external and internal morphology of these teeth was described by the collection of published case reports. Materials and Methods. Six electronic databases were accessed to collect case reports dealing with fourrooted maxillary first molars, as well as population-based cone-beam computed tomography (CBCT) studies. Afterward, the publications were selected according to predefined inclusion/exclusion criteria and evaluated using the Joanna Briggs Institute Critical Appraisal tool. The teeth of the chosen case reports were then independently analyzed by two dental professionals according to different dental classifications. Furthermore, the population studies were meta-analyzed to calculate the global and regional prevalence of four-rooted maxillary molars. Results. Included were forty-nine population-based CBCT studies containing 26663 maxillary first molars. Upon these data, the global incidence of four-rooted maxillary molars was meta-analytically determined as $0.047 \%$ (95\%-CI:0.011-0.103\%). In combination with the case reports, it was pointed out that this anomaly is distributed worldwide. Furthermore, forty-eight case reports were included containing fifty-three maxillary molars with four roots. The analyzed teeth exhibited Versiani's pulpal chamber floor Types A and B. The majority of four-rooted maxillary first molars were classified as Type I regarding Christie's configuration. But, also $7.54 \%$ of the altered teeth could not be described by this classification. $62.34 \%$ exhibited four root canals, but also variations with five, six, or seven canals were identified. Furthermore, a significant difference was found in the occurrence rate between male and female patients. Conclusion. Due to the worldwide occurrence, dental professionals should be aware of this rare anomaly to avoid treatment errors, especially during endodontic or surgical therapies.
\end{abstract}

\section{Introduction}

For centuries, anatomists have investigated dental anatomy to describe each human tooth by determining its coronal and radicular structures. Human maxillary first molars are generally characterized to possess four or five cusps (the additional mesiolingual cusp of Carabelli) and three roots $[1,2]$. These roots were designated due to their anatomical position as mesiobuccal, distobuccal, and palatal root, usually exhibiting four root canals (mesiobuccal root with a second canal, whereas the other roots commonly contain only one canal) $[3,4]$. However, numerous studies and case reports also presented maxillary first molars with various anatomical alterations resulting in an enormous diversity in the number of roots and canals. For example, rare cases of single, two-, or even five-rooted maxillary first molars were described [3,5-7] as well as three-rooted versions with multiple canals [8-10].

Another rare aberration was described by Thews et al. 1979 [11], who identified radiographically four separate 
roots during endodontic treatment. This unlikely morphological anomaly was classified upon the radicular shape and the degree of root separation by Christie et al. in 1991 [12]. Their characterization was based mainly on studying endodontic treated or extracted maxillary molars and identified three different radicular configurations. Type I maxillary molars have widely divergent, long, and tortuous palatal roots with "cow-horn" shaped buccal roots. Type II maxillary molars have four, shorter, parallel running roots with blunt apices. By definition, "a type III maxillary molar is constricted in root morphology with the mesiobuccal, mesiopalatal, and distopalatal canal encaged in a web of root dentin" [12]. Furthermore, Baratto-Filho et al. in 2002 demonstrated an endodontic case with fused mesiobuccal and mesiopalatal roots and suggested an additional class IV [13] (Figure 1).

However, Versiani et al. in 2012 indicated by studying four-rooted maxillary second molars with micro-CT the Christie's configuration as not feasible, because fusions might occur on a different root level [14]. Therefore, they defined a classification introducing a new type III with less divergent and short palatal roots along with widely divergent buccal roots. In addition, Christie's Types II and III were combined $[12,14]$. However, another classification for this very rare anatomical anomaly was designed by naming the additional palatal root as radix mesio- or distolingualis based on its direct affinity to the pronounced part of the crown [15]. Moreover, Carlsen and Alexandersen described maxillary molars exhibiting three buccal roots and characterized the additional radicular structure as radix paramolaris [16] (Figure 1).

Interestingly, all mentioned classifications depended on the investigation of the second or even third maxillary molars and were commonly used for the description of maxillary first molars without any scientific proof $[12,14,15]$. However, due to the rarity of four-rooted maxillary first molars, no anatomical study could be found in dental literature [3]. Therefore, this study systematically collected case reports on maxillary first molars with four roots to study their specific anatomy. In addition, population-based cone-beam computed tomographic (CBCT) studies were analyzed to assess the unknown worldwide prevalence and distribution of these teeth.

\section{Materials and Methods}

2.1. Literature Search Strategy. This systematic review, case report collection, and meta-analysis followed the Preferred Reporting Items for Systematic Reviews and Meta-Analyses (PRISMA) [17]. A literature search was conducted between August 2019 and November 2019 by both authors. Six electronic databases were searched (Google Scholar, PubMed, BASE (Bielefeld Academic Search Engine), SciELO (Scientific Electronic Library Online), AJOL (African Journals OnLine), and DHBD (Circumpolar Health Bibliographic Database)) for population-based studies using CBCT imaging for the investigation of maxillary first molars root anatomy according to specific terms (MeSH terms: "maxillary first molar" "cone-beam computed tomography"). Publications in Chinese, English, German, Italian, Japanese, Portuguese, Russian, Spanish, and Turkish were identified. Afterward, the references for these studies were hand-searched. The selection procedure of the records followed a three-step evaluation. At first, titles and abstracts were accessed and characterized as relevant or irrelevant in agreement with predefined inclusion and exclusion criteria (Suppl. Table 1). Afterward, the full texts (if available) of the selected articles were evaluated according to the mentioned criteria. The resulting articles were critically assessed and evaluated independently by both authors based on the JBI questionnaire [18]. Furthermore, few authors were contacted via e-mail to receive missing information on their studies according to the inclusion criteria and the JBI questions.

Additionally, the mentioned databases were accessed to identify case reports concerning maxillary molars with four roots (MeSH terms: "maxillary first molar" "four roots," "maxillary first molar" "two palatal roots," "maxillary first molar" "Radix mesiolingualis," "maxillary first molar" "Radix distolingualis"). Publications in Chinese, English, Farsi, Portuguese, Spanish, and Turkish were identified. Afterward, the reference lists of the identified case reports were hand-searched. As mentioned above, at first, the titles and abstracts were evaluated according to predefined inclusion and exclusion criteria (Suppl. Table 1). Second, the available full texts were analyzed upon these criteria. Finally, both authors defined the case report teeth upon Christie's radicular configuration regarding the published radiographs [12]. Afterward, when available, the intraoperative photographs of the teeth were classified according to Versiani's pulpal chamber floor type [14] (Figure 2). Disagreements were resolved through consensus.

2.2. Statistical Analysis. The summary of the selected (population-based CBCT) studies and the calculation of the pooled proportion of teeth with 4 roots were carried out by a random-effects model with inverse-variance weights. Since some CBCT studies showed no record of upper first molars with 4 roots, the proportions of individual studies were first transformed for the calculation of the pooled proportion (Freeman-Tukey Double Arcsine Transformation). The graphical representation of the proportions of the individual studies and the fraction pooled share with a 95\% confidence interval was carried out with a forest plot. In addition, the dispersion of the studies was illustrated in a funnel plot in which the number of teeth of each study was plotted against the proportion of teeth with 4 root canals. Furthermore, Cohen's kappa was calculated to quantify the degree of compliance of two assessors in answering the JBI questions. All statistical tests were carried out on two sides at the significance level 0.05. Stata/IC 16.1 for Unix (StataCorp 4905 Lakeway Drive, College Station, TX 77845, USA) was used for data preparation and analysis. 

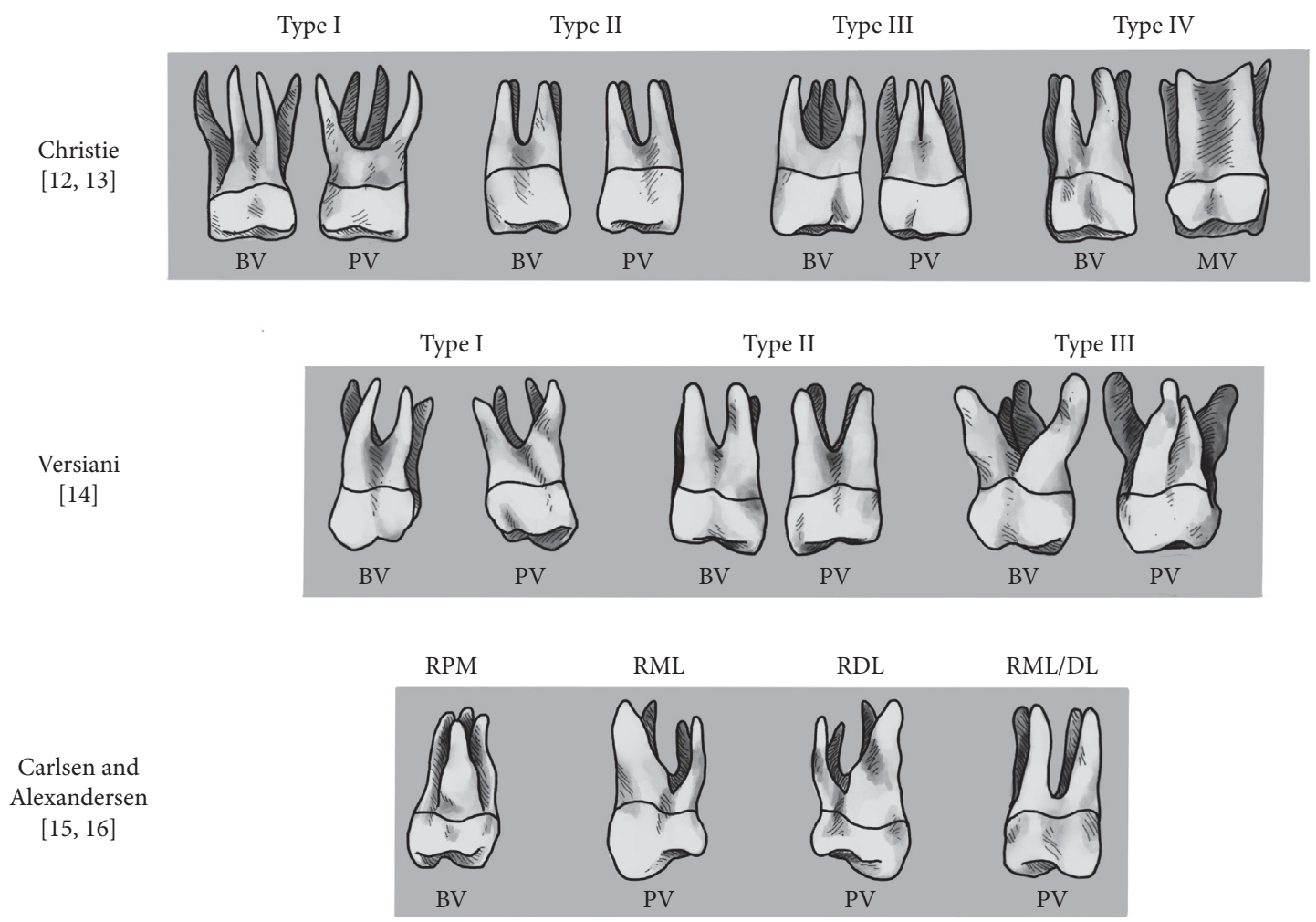

FIGURE 1: Schematic drawing of radicular classifications of four-rooted maxillary molars modified from [12-16]. Christie classified fourrooted maxillary molars in Type I to Type III based on the radicular shape and the degree of root separation [12]. Type IV was added by Baratto-Filho [13] with a fused mesiobuccal and mesiopalatal root. Versiani's modified radicular classification [14]. Carlsen and Alexandersen defined and named additional radicular structures upon their buccal or palatal location and according to their affinity to the dental crown. BV:buccal view, PV:palatal view, MV:mesial view, RPM:radix paramolaris, RML:radix mesiolingualis, RDL:radix distolingualis, RML/DL:radix mesiolingualis/distolingualis.

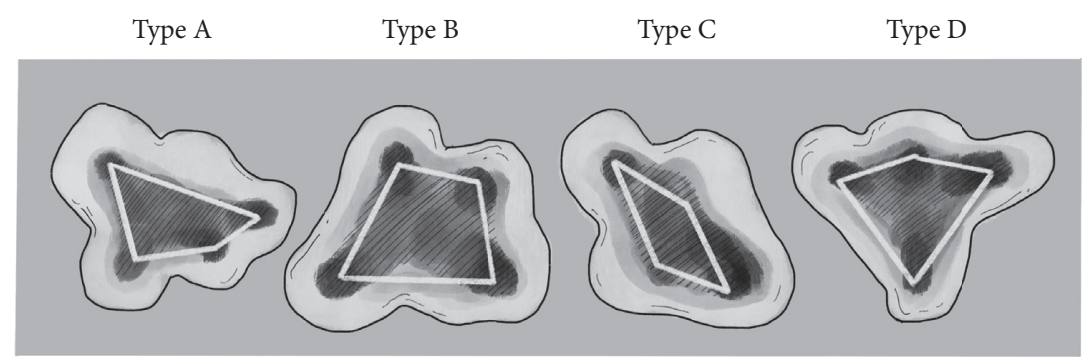

Figure 2: Schematic drawing of Versiani's configuration of canal orifices in four-rooted maxillary second molars [14]. The orifices were classified in relation to the pulpal chamber floor as Type A (irregular quadrilateral-shaped), Type B (trapezoid-shaped), Type C (lozengeshaped), and Type D (kite-shaped).

\section{Results}

3.1. Included Studies. The electronic and manual search identified 117 relevant studies for the population-based CBCT studies. Sixty-eight studies were excluded by accessing the title, abstract, and/or full text (Figure 3). The JBI questions [18] Q5 and Q9 were considered not applicable, and Q6 was eliminated due to the predefined CBCT technique like previous dental studies described [19]. The results of the Cohen kappa interrater reliability for the publications investigated by the JBI questionnaire were 0.883 (Q1), 0.795 (Q2), 0.979 (Q4), 0.645 (Q7), and $0.764(\mathrm{Q} 8)$. The resulting
54 studies had an average score of $77.2 \%$. Five studies were excluded due to their low score $(\leq 50 \%)$, whereas 38 papers had a high $(\geq 75 \%)$ and 11 a moderate $(\geq 50 \%)$ score. The finally selected 49 studies included 26663 investigated maxillary first molars (Table 1).

From 140 selected case reports concerning four-rooted maxillary first molars, 93 publications were excluded based on the predefined inclusion and exclusion criteria. One population-based CBCT study also documented a micro-CT of a four-rooted molar and was included (Figure 3). The finally selected 48 studies included 53 four-rooted maxillary molars (Table 2). In combination with additional 


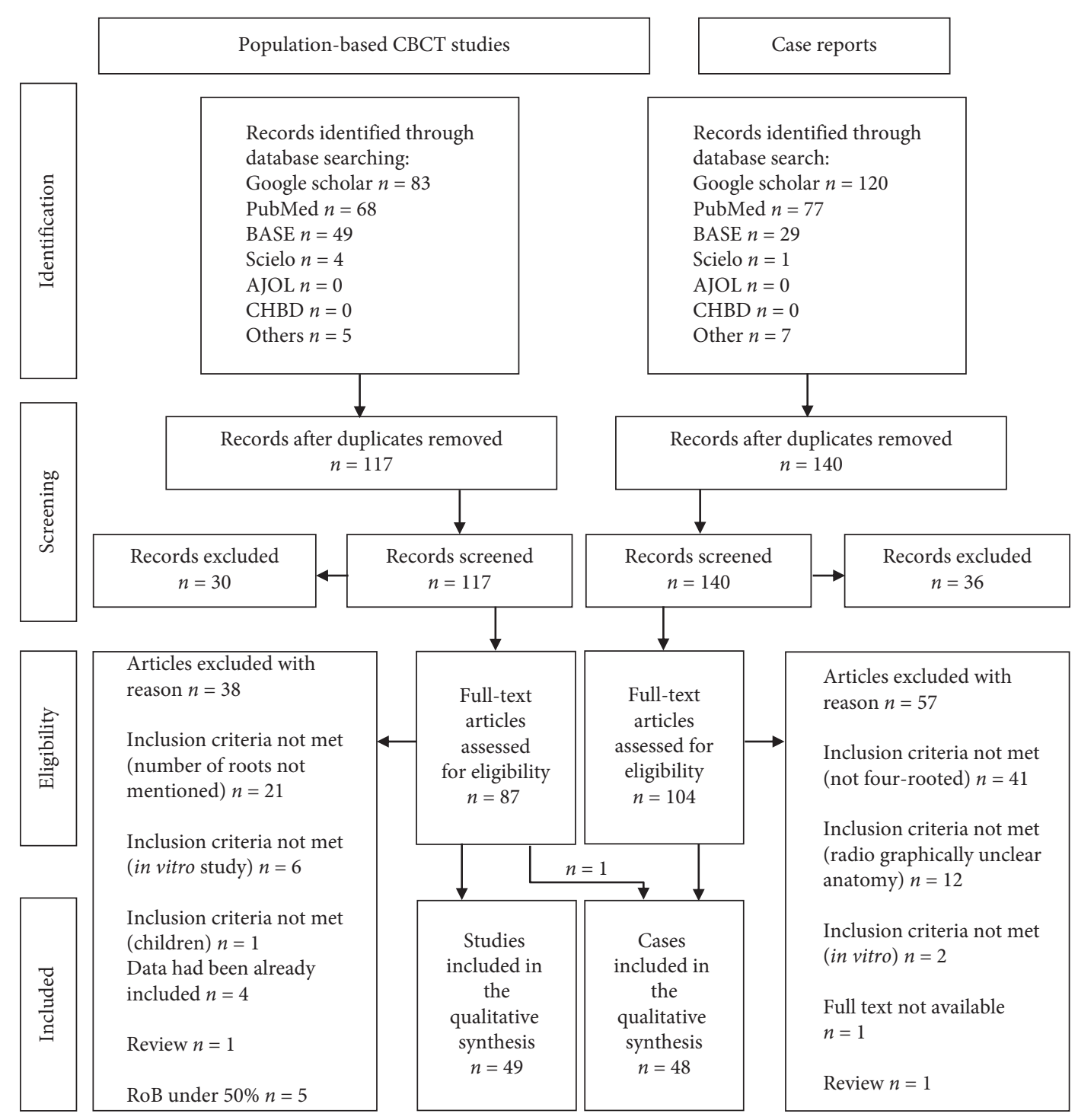

FIgURE 3: Flowchart summarizing the search strategy and results.

information in the CBCT studies, the sample size of the analyzed teeth could be increased. So, 75 maxillary first molars with four roots could be investigated regarding their amount of root canals and 67 teeth to study the gender of the patients. On the other hand, the radicular subtype (46 teeth), Versiani's pulpal chamber floor type (33 teeth), and the distinction of the right or left molar (49 teeth) had a less amount of studied subjects due to missing data in the case reports.

3.2. Global Distribution. Four-rooted maxillary molars were identified worldwide in population-based CBCT studies and case reports (Figure 4). The composed prevalence (95\% CI) of the analyzed 26663 teeth was calculated as $0.047 \%$ (0.011-0.103\%) (Table 1). The highest prevalence was metaanalytically found in the Greek-Turkish population with $0.804 \%(0.255-1.609 \%)$. Most of the case reports (43.4\%) were documented in India in contrast to the moderate prevalence rate of $0.024 \%(0-0.249 \%)$ (Figure 4, Suppl.
Table 2.1-14). The funnel plot demonstrated that four of the 49 included studies were outside the margins of the $95 \%$ confidence interval. This was in the expected proportion of $95 \%$ of studies between the curves, resulting in no risk of bias (Figure 5).

3.3. Anatomical Description of Four-Rooted Maxillary Molars. The analysis of the case reports (Table 2), according to Christie's radicular structure, demonstrated that $37.7 \%$ of the authors used this classification. By using Christie's accurate description for the radiographic identification, we characterized $52.83 \%$ as Type I, $18,87 \%$ as Type II, $18,78 \%$ as Type III, 1 , and $88 \%$ as Type IV, and $7.54 \%$ could not be described by this classification (Table 3). Versiani's introduced pulpal chamber floor type was only used by Magnucki et al. in 2018 [61]. Our analysis classified the geometrical location of the root canal orifices in $45.45 \%$ as Type A (irregular quadrilateral), $51.52 \%$ as Type B (trapezoid), and $3.03 \%$ as Type $D$ (kite-shaped). Type C could not be found in 
TABle 1: Analysis of the population-based CBCT studies.

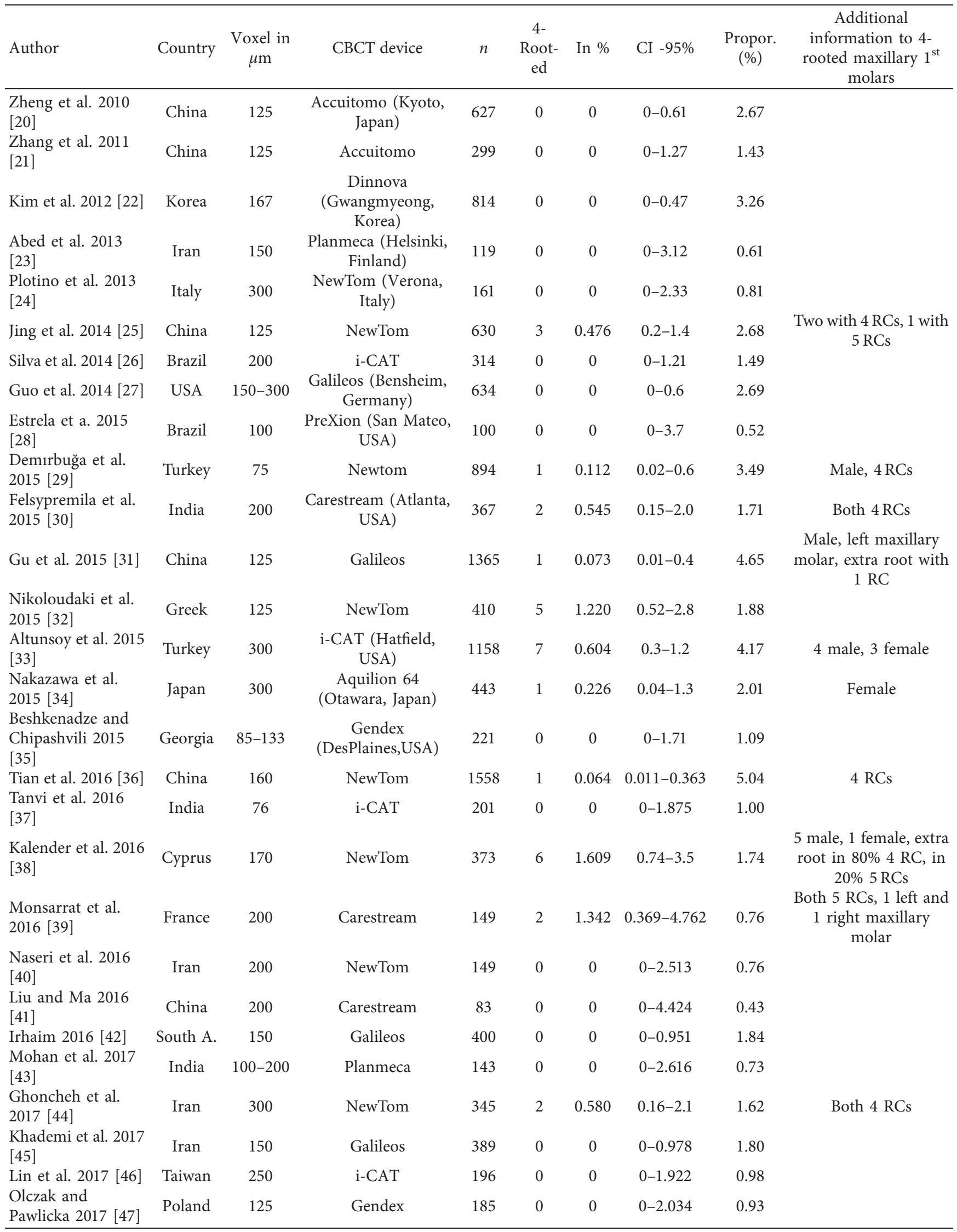


TABLe 1: Continued.

\begin{tabular}{|c|c|c|c|c|c|c|c|c|c|}
\hline Author & Country & $\begin{array}{l}\text { Voxel in } \\
\qquad \mu \mathrm{m}\end{array}$ & CBCT device & $n$ & $\begin{array}{c}4- \\
\text { Root- } \\
\text { ed }\end{array}$ & In $\%$ & CI -95\% & $\begin{array}{l}\text { Propor. } \\
\text { (\%) }\end{array}$ & $\begin{array}{c}\text { Additional } \\
\text { information to } 4- \\
\text { rooted maxillary } 1^{\text {st }} \\
\text { molars }\end{array}$ \\
\hline $\begin{array}{l}\text { Pérez-Heredia et al. } \\
2017 \text { [48] }\end{array}$ & Spain & 180 & Carestream & 142 & 0 & 0 & $0-2.634$ & 0.72 & \\
\hline $\begin{array}{l}\text { Ghobashy et al. } \\
2017 \text { [49] }\end{array}$ & Egypt & 133 & $\begin{array}{l}\text { Cranex (Tuusula, } \\
\text { Finland) }\end{array}$ & 605 & 0 & 0 & $0-0.631$ & 2.59 & \\
\hline $\begin{array}{l}\text { Wang et al. } 2017 \\
\text { [50] }\end{array}$ & China & 200 & Planmeca & 953 & 2 & 0.210 & $0.06-0.8$ & 3.65 & $\begin{array}{c}1 \text { with } 4 \text { RCs, } 2 \text { left } \\
\text { molars }\end{array}$ \\
\hline $\begin{array}{l}\text { Al-Shehri et al. } \\
2017 \text { [51] }\end{array}$ & Saudi-A. & 300 & Different & 351 & 0 & 0 & $0-1.083$ & 1.65 & \\
\hline $\begin{array}{l}\text { Al-Kadhim et al. } \\
2017 \text { [52] }\end{array}$ & Malaysia & $300-600$ & Accuitomo & 421 & 0 & 0 & $0-0.90$ & 1.92 & \\
\hline $\begin{array}{l}\text { Ogawa and Seki } \\
2017 \text { [53] }\end{array}$ & Japan & 160 & Planmeca & 240 & 1 & 0.417 & $0.07-2.3$ & 1.17 & \\
\hline $\begin{array}{l}\text { Zhang et al. } 2017 \\
\text { [54] }\end{array}$ & China & 150 & NewTom & 1008 & 0 & 0 & $0-0.38$ & 3.80 & \\
\hline $\begin{array}{l}\text { Martins et al. } 2018 \\
\text { [55] }\end{array}$ & Portugal & 200 & Planmeca & 567 & 0 & 0 & $0-0.67$ & 2.46 & \\
\hline $\begin{array}{l}\text { Razumova et al. } \\
2018 \text { [56] }\end{array}$ & Russia & 300 & $\begin{array}{c}\text { 3D eXam } \\
\text { (Hatfield,USA) }\end{array}$ & 410 & 0 & 0 & $0-0.93$ & 1.88 & \\
\hline $\begin{array}{l}\text { Martins et al. } 2018 \\
\text { [57] }\end{array}$ & Worldw. & $75-250$ & Different & 5000 & 9 & 0.180 & $0.10-0.3$ & 8.59 & $\begin{array}{c}2 \text { with } 4 \text { RCs, } 7 \text { with } \\
5 \mathrm{RCs}\end{array}$ \\
\hline $\begin{array}{l}\text { Salem et al. } 2018 \\
\text { [58] }\end{array}$ & Egypt & 150 & Planmeca & 138 & 0 & 0 & $0-2.71$ & 0.70 & \\
\hline $\begin{array}{l}\text { Arbildo Villalta } \\
2018 \text { [59] }\end{array}$ & Peru & 125 & Planmeca & 168 & 0 & 0 & $0-2.235$ & 0.85 & \\
\hline $\begin{array}{l}\text { Ratanajirasut et al. } \\
2018 \text { [60] }\end{array}$ & Thailand & 100 & Accuitomo & 476 & 1 & 0.210 & $0.04-1.2$ & 2.13 & $4 \mathrm{RCs}$ \\
\hline Salzmann 2018 [61] & Austria & 80 & Accuitomo & 147 & 0 & 0 & $0-2.55$ & 0.75 & \\
\hline Pan et al. 2019 [62] & Malysia & 250 & 3D eXam & 344 & 0 & 0 & $0-1.10$ & 1.62 & \\
\hline $\begin{array}{l}\text { Candeiro et al. } 2019 \\
\text { [63] }\end{array}$ & Brazil & 125 & Prexion & 700 & 2 & 0.286 & $0.08-1.0$ & 2.90 & \\
\hline $\begin{array}{l}\text { Mohara et al. } 2019 \\
\text { [64] }\end{array}$ & Brazil & 125 & Accuitomo & 328 & 0 & 0 & $0-1.16$ & 1.55 & \\
\hline $\begin{array}{l}\text { Kewalramani et al. } \\
2019 \text { [65] }\end{array}$ & India & 180 & Carestream & 600 & 0 & 0 & $0-0.64$ & 2.57 & \\
\hline $\begin{array}{l}\text { Popović et al. } 2019 \\
\text { [66] }\end{array}$ & Serbia & 160 & Galileos & 138 & 0 & 0 & $0-2.71$ & 0.70 & \\
\hline $\begin{array}{l}\text { Mashyakhy and } \\
\text { gambarini } 2019 \\
{[67]}\end{array}$ & Saudi-A. & 250 & Accuitomo & 354 & 0 & 0 & $0-1.07$ & 1.66 & \\
\hline $\begin{array}{l}\text { Tzeng et al. } 2019 \\
\text { [68] }\end{array}$ & Taiwan & 250 & i-CAT & 846 & 1 & 0.118 & $0.02-0.7$ & 3.35 & Male, 5 RCs, left \\
\hline World & \multicolumn{3}{|c|}{ Overall random pooled $*$} & 26663 & 47 & 0.047 & $0.011-0.103$ & 100 & \\
\hline
\end{tabular}

CBCT:cone-beam computed tomographic, Propor.:proportion, RC:root canal, Saudi-A.:Saudi Arabia, South A.:South Africa, Worldw.:worldwide.

the investigated case reports (Table 3 ). The majority of the analyzed teeth (combined CBCT studies and case reports, a sample size of 78 teeth) demonstrated four (62.34\%) or five $(27.27 \%)$ root canals. But also six $(9.09 \%)$ or even seven $(1.30 \%)$ root canals were described (Table 3$)$. No statistical difference could be found between the left (50.00\%) and right $(50.00 \%)$ of maxillary first molars with four roots. Of the 68 teeth, where the sex of the patient was mentioned, $60.29 \%$ were males, and $39.71 \%$ were females. This difference was significant (Table 3). Coronal anomalies or enamel pearls in addition to the morphological alteration of four roots were documented in case reports in $15.1 \%$.

\section{Discussion}

The knowledge of oral anatomy, its anomalies, and their frequencies is fundamental for successful dental therapies. Therefore, this systematic review assessed the prevalence of maxillary first molars with four roots in humans and documented a very low global occurrence rate of $0.047 \%$. Nevertheless, these morphologically altered teeth were found worldwide in documented case reports and population studies, except for Eastern Europe, Australia, and mostly Africa. Probably, a higher study activity in the field of dental anatomy could fill these areas, as it is mainly the lack 
TABLe 2: Analysis of the case reports.

\begin{tabular}{|c|c|c|c|c|c|c|c|c|c|c|c|}
\hline \multirow[b]{2}{*}{ Author } & \multirow[b]{2}{*}{ Country } & \multirow[b]{2}{*}{ Age } & \multirow[b]{2}{*}{ Sex } & \multirow{2}{*}{$\begin{array}{l}\text { Tooth } \\
\text { number }\end{array}$} & \multicolumn{4}{|c|}{ Root canals } & \multirow{2}{*}{$\begin{array}{c}\text { Christies's } \\
\text { radicular type } \\
{[12,13]}\end{array}$} & \multirow{2}{*}{$\begin{array}{l}\text { Versiani's } \\
\text { pulpal floor } \\
\text { type [14] }\end{array}$} & \multirow[b]{2}{*}{ Additional information } \\
\hline & & & & & $M B$ & $D B$ & $M P$ & $D P$ & & & \\
\hline $\begin{array}{l}\text { Thews et al. } 1979 \\
\text { [11] }\end{array}$ & USA & 21 & $\mathrm{M}$ & n.m. & 1 & 1 & 1 & 1 & n.m. (I) & - & $\begin{array}{l}\text { Single, enlarged lingual } \\
\text { cusp }\end{array}$ \\
\hline $\begin{array}{l}\text { Stabholz and } \\
\text { Friedman } 1983 \\
{[69]}\end{array}$ & Israel & 13 & $\mathrm{~F}$ & 16 & 2 & 1 & 1 & 1 & n.m. (III) & B & \multirow[t]{3}{*}{$\begin{array}{c}\text { Unusual configuration } \\
\text { of the crown }\end{array}$} \\
\hline $\begin{array}{l}\text { Wong et al. } 1991 \\
{[70]}\end{array}$ & USA & 22 & $\mathrm{M}$ & 26 & 2 & 1 & 2 & 1 & n.m. (III) & - & \\
\hline $\begin{array}{l}\text { Christie et al. } 1991 \\
\text { [12] }\end{array}$ & Canada & n.m. & $\mathrm{F}$ & 16 & 1 & 1 & 1 & 1 & I & B & \\
\hline $\begin{array}{l}\text { Jacobsen and Nii } \\
1994[71]\end{array}$ & USA & 25 & $\mathrm{M}$ & 26 & 2 & 1 & 1 & 1 & n.m. (III) & & \multirow[t]{2}{*}{ Presented case 3} \\
\hline Di Fiore 1999 [72] & USA & 31 & M & 16 & 1 & 1 & 1 & 1 & II & $\mathrm{B}$ & \\
\hline $\begin{array}{l}\text { Baratto-Filho et al. } \\
2002[13]\end{array}$ & Japan & 38 & $\mathrm{~F}$ & 16 & 1 & 1 & 1 & 1 & I & - & Presented case 1 \\
\hline $\begin{array}{l}\text { Maggiore et al. } \\
2002[73]\end{array}$ & USA & 19 & M & 26 & 2 & 1 & 2 & 1 & n.m. (III) & - & Third palatal canal \\
\hline $\begin{array}{l}\text { Barbizam et al. } \\
2004 \text { [5] }\end{array}$ & Brazil & 35 & $\mathrm{M}$ & 26 & 1 & 1 & 1 & 1 & I & - & \multirow[t]{6}{*}{ Presented case 1} \\
\hline $\begin{array}{l}\text { Nahmias and Bery } \\
2006[74]\end{array}$ & Canada & 58 & $\mathrm{~F}$ & 26 & 1 & 1 & 1 & 1 & n.m. (I) & - & \\
\hline Adanir 2007 [75] & Turkey & 31 & $\mathrm{M}$ & 16 & 2 & 2 & 1 & 1 & n.m. (II) & A & \\
\hline Ravishanker and & & & & & & & & & & & \\
\hline $\begin{array}{l}\text { Prashanthi } 2008 \\
\text { [76] }\end{array}$ & India & 25 & $\mathrm{~F}$ & 26 & 1 & 1 & 1 & 1 & n.m. (I) & - & \\
\hline $\begin{array}{l}\text { Gandhi and } \\
\text { Madan } 2009 \text { [77] }\end{array}$ & India & 50 & $\mathrm{M}$ & 26 & 1 & 1 & 1 & 1 & n.m. (II) & $\mathrm{B}$ & \\
\hline $\begin{array}{l}\text { Tomazinho et al. } \\
2010 \text { [78] }\end{array}$ & Brazil & 32 & $\mathrm{M}$ & 26 & 2 & 2 & 1 & 1 & I & B & \multirow[t]{5}{*}{$\begin{array}{l}\mathrm{MB} \text { and } \mathrm{DB} \mathrm{RC} \text { : } \\
\text { Vertucci Class II }\end{array}$} \\
\hline $\begin{array}{l}\text { Salapoor and } \\
\text { Mollashahi } 2010 \\
\text { [79] }\end{array}$ & Iran & 40 & $\mathrm{~F}$ & n.m. & 1 & 1 & 1 & 1 & n.m. (II) & - & \\
\hline He et al. 2010 [80] & China & 35 & M & 16 & 1 & 1 & 1 & 1 & n.m. (II) & A & \\
\hline $\begin{array}{l}\text { Chakradhar Raju } \\
\text { et al. } 2010 \text { [81] }\end{array}$ & India & 24 & M & 26 & 1 & 1 & 1 & 1 & I & B & \\
\hline & India & 21 & M & 16 & 1 & 1 & 1 & 1 & I & - & \\
\hline $\begin{array}{l}\text { Moghaddas and } \\
\text { Tabari } 2010 \text { [82] }\end{array}$ & Iran & 41 & $\mathrm{~F}$ & 26 & 1 & 1 & 1 & 1 & n.m. (I) & - & $\begin{array}{l}\text { Enamel pearl, } \\
\text { hemisection }\end{array}$ \\
\hline $\begin{array}{l}\text { Kottoor et al. } 2011 \\
{[83]}\end{array}$ & India & 42 & $\mathrm{M}$ & 16 & 1 & 1 & 1 & 1 & n.m. (III) & A & 2 fused palatal roots \\
\hline $\begin{array}{l}\text { Singh et al. } 2011 \\
{[84]}\end{array}$ & India & 21 & $\mathrm{~F}$ & 16 & 1 & 1 & 1 & 1 & n.m. (I) & A & \multirow[t]{3}{*}{ Presented case 1} \\
\hline $\begin{array}{l}\text { Reddy et al. } 2011 \\
\text { [85] }\end{array}$ & India & 45 & $\mathrm{M}$ & 16 & 1 & 1 & 1 & 1 & n.m. (I) & $\mathrm{B}$ & \\
\hline Du et al. 2011 [86] & China & 21 & $\mathrm{~F}$ & 26 & 2 & 1 & 1 & 1 & n.m. (III) & $\mathrm{B}$ & \\
\hline $\begin{array}{l}\text { Madhuram et al. } \\
2012 \text { [87] }\end{array}$ & India & 27 & $\mathrm{~F}$ & n.m. & 1 & 1 & 1 & 1 & n.m. (III) & - & \multirow{3}{*}{$\begin{array}{c}\text { Pronounced Carabelli } \\
\text { tubercle } \\
\text { Second mesiobuccal } \\
\text { root, } 16 \text { with identical } \\
\text { morphology }\end{array}$} \\
\hline $\begin{array}{l}\text { Kottoor et al. } 2012 \\
{[88]}\end{array}$ & India & 23 & $\mathrm{M}$ & 26 & 1 & 1 & 1 & 1 & n.d. & A & \\
\hline & India & 23 & $\mathrm{M}$ & 16 & n.m & n.m. & n.m. & n.m. & n.d. & - & \\
\hline $\begin{array}{l}\text { Mathew et al. } 2013 \\
\text { [89] }\end{array}$ & India & 35 & M & 16 & 1 & 1 & 1 & 1 & I & - & \multirow{4}{*}{$\begin{array}{l}\text { Second mesiobuccal } \\
\text { root }\end{array}$} \\
\hline $\begin{array}{l}\text { Rajalbandi et al. } \\
2013 \text { [90] }\end{array}$ & India & 42 & $\mathrm{M}$ & 26 & 1 & 1 & 1 & 1 & I & $\mathrm{B}$ & \\
\hline $\begin{array}{l}\text { Yesidal Yeter et al. } \\
2013[91]\end{array}$ & Turkey & 28 & M & 16 & 2 & 2 & 1 & 1 & n.d. & A & \\
\hline $\begin{array}{l}\text { Aggarwal et al. } \\
2013 \text { [92] }\end{array}$ & India & 24 & $\mathrm{M}$ & 26 & 1 & 1 & 1 & 1 & I & A & \\
\hline
\end{tabular}


TABLE 2: Continued.

\begin{tabular}{|c|c|c|c|c|c|c|c|c|c|c|c|}
\hline \multirow[b]{2}{*}{ Author } & \multirow[b]{2}{*}{ Country } & \multirow[b]{2}{*}{ Age } & \multirow[b]{2}{*}{ Sex } & \multirow{2}{*}{$\begin{array}{c}\text { Tooth } \\
\text { number }\end{array}$} & \multicolumn{4}{|c|}{ Root canals } & \multirow{2}{*}{$\begin{array}{c}\text { Christies's } \\
\text { radicular type } \\
{[12,13]}\end{array}$} & \multirow{2}{*}{$\begin{array}{l}\text { Versiani’s } \\
\text { pulpal floor } \\
\text { type [14] }\end{array}$} & \multirow[b]{2}{*}{ Additional information } \\
\hline & & & & & $M B$ & $D B$ & $M P$ & $D P$ & & & \\
\hline \multirow[t]{2}{*}{$\begin{array}{l}\text { Ghani et al. } 2013 \\
\text { [93] }\end{array}$} & India & 34 & $\mathrm{~F}$ & 16 & 1 & 1 & 1 & 1 & I & B & $\begin{array}{l}\text { End. retreatment, both } \\
\text { teeth fused MB\&MP } \\
\text { roots }\end{array}$ \\
\hline & India & 34 & $\mathrm{~F}$ & 26 & 1 & 1 & 1 & 1 & I & B & \\
\hline $\begin{array}{l}\text { Sharma et al. } 2014 \\
\text { [94] }\end{array}$ & India & 31 & $\mathrm{~F}$ & 16 & 1 & 2 & 1 & 1 & n.d. & $\mathrm{D}$ & $\begin{array}{l}\text { Fused MB\&MP and } \\
\text { DB\&DP roots }\end{array}$ \\
\hline $\begin{array}{l}\text { Kararia et al. } 2014 \\
\text { [95] }\end{array}$ & India & 20 & $\mathrm{~F}$ & 26 & 1 & 2 & 1 & 1 & I & B & $\begin{array}{c}\text { Endodontic } \\
\text { retreatment, DB canal: } \\
\text { Vertucci Class II }\end{array}$ \\
\hline $\begin{array}{l}\text { Shah and Jadhav } \\
2014 \text { [96] }\end{array}$ & India & 38 & $\mathrm{~F}$ & 16 & 1 & 1 & 1 & 1 & n.m. (II) & B & $\begin{array}{l}\text { Crown with four } \\
\text { palatal cusps }\end{array}$ \\
\hline $\begin{array}{l}\text { Sánchez-Salas } \\
\text { et al. } 2014 \text { [97] }\end{array}$ & Mexico & 31 & M & 26 & 1 & 1 & 1 & 1 & I & B & \\
\hline Shrestha 2015 [98] & Nepal & 58 & $\mathrm{~F}$ & 26 & 2 & 1 & 1 & 1 & II & A & $\begin{array}{c}\text { Presented case 1, End. } \\
\text { Retreatment }\end{array}$ \\
\hline $\begin{array}{l}\text { Asghari et al. } 2015 \\
\text { [99] }\end{array}$ & Iran & 21 & $\mathrm{~F}$ & 16 & 1 & 1 & 1 & 1 & I & B & \\
\hline $\begin{array}{l}\text { Wu and Wu } 2015 \\
{[100]}\end{array}$ & China & 29 & M & 26 & 1 & 1 & 1 & 1 & n.m. (II) & B & \\
\hline \multirow{4}{*}{$\begin{array}{l}\text { Gu et al. } 2015 \text { [31] } \\
\text { Deepa et al. } 2016 \\
\text { [101] }\end{array}$} & China & 37 & M & 16 & 1 & 1 & 1 & 1 & n.m. (I) & $\mathrm{v}$ & $\begin{array}{c}\text { Enamel pearl, } \\
\text { pronounced cusp of } \\
\text { Carabelli }\end{array}$ \\
\hline & China & n.a. & n.a. & 16 & 1 & 2 & 1 & 2 & n.m. (IV) & - & Fused MB\&MP roots \\
\hline & India & 41 & $\mathrm{~F}$ & 16 & 1 & 1 & 1 & 1 & n.m. (I) & - & CBCT after extraction \\
\hline & India & n.m. & n.m. & n.m. & 1 & 1 & 1 & 1 & I & A & $\begin{array}{l}\text { End. Retreatment, DB } \\
\text { canal: Vertucci II }\end{array}$ \\
\hline $\begin{array}{l}\text { Tao et al. } 2016 \\
{[102]}\end{array}$ & China & 10 & $\mathrm{~F}$ & 16 & 1 & 1 & 1 & 1 & n.m. (II) & A & $\begin{array}{l}\text { Three well-developed } \\
\text { lobulated palatal cusps }\end{array}$ \\
\hline $\begin{array}{l}\text { Nayak et al. } 2016 \\
\text { [103] }\end{array}$ & India & 24 & M & 16 & 1 & 1 & 1 & 1 & I & A & \\
\hline $\begin{array}{l}\text { Barreto and Lins } \\
2016[104]\end{array}$ & Brazil & 28 & M & 26 & 1 & 1 & 1 & 1 & n.m. (I) & - & $\begin{array}{c}\text { MB canal: Vertucci } \\
\text { Type II }\end{array}$ \\
\hline $\begin{array}{l}\text { Vázquez and } \\
\text { Llácer } 2016 \text { [105] }\end{array}$ & Spain & 46 & M & 16 & 2 & 1 & 1 & 1 & n.m. (I) & A & \\
\hline $\begin{array}{l}\text { Rodrigues et al. } \\
2016 \text { [106] }\end{array}$ & Brazil & 23 & $\mathrm{~F}$ & 16 & 3 & 2 & 1 & 1 & n.m. (III) & A & \\
\hline $\begin{array}{l}\text { Cao et al. } 2017 \\
{[107]}\end{array}$ & China & 30 & M & 16 & 2 & 1 & 1 & 1 & n.m. (II) & B & \\
\hline $\begin{array}{l}\text { Magnucki et al. } \\
2018 \text { [108] }\end{array}$ & Germany & 51 & M & 26 & 2 & 1 & 1 & 1 & I & A & $\begin{array}{c}2 \text { enamel pearls, MB } \\
\text { RC: Vertucci II }\end{array}$ \\
\hline $\begin{array}{l}\text { Meena and Hasija } \\
2018 \text { [109] }\end{array}$ & India & 27 & $\mathrm{~F}$ & 16 & 1 & 1 & 1 & 1 & n.m. (III) & - & \\
\hline $\begin{array}{l}\text { Schryvers et al. } \\
2018 \text { [110] }\end{array}$ & Belgium & 44 & M & 26 & 1 & 1 & 1 & 1 & I & A & \\
\hline $\begin{array}{l}\text { Sriganesh and } \\
\text { Priyan } 2019 \text { [111] }\end{array}$ & India & 18 & $\mathrm{~F}$ & 26 & 2 & 1 & 1 & 1 & n.m. (III) & - & \\
\hline
\end{tabular}

DB :distobuccal, DP:distopalatal, End.:endodontic, F:female, M: male, MB:mesiobuccal, MP:mesiopalatal, n.d.:not defined, n.m. :not mentioned, RC:root canal.

of publication (e.g., 250 investigated teeth in Australia), which led to these unaddressed geographic regions. Population-based CBCT studies with high amounts of sample size can identify even very rare anatomical anomalies and are therefore a suitable scientific tool $[4,19,57]$. Besides an appropriate number of studied teeth, a reproducible methodology and detailed description of demographic factors are recommended as a guideline for these studies
$[4,19]$. The present study linked the occurrence of fourrooted maxillary first molars with regionally subgrouped populations based on the demonstrated association of rare morphological variations and ethnicity in dental literature $[19,112]$. The highest occurrence rate was found in the Greek-Turkish population with a significantly higher prevalence than in all other populations. The lowest rates were identified in Eastern Europe and on the American 


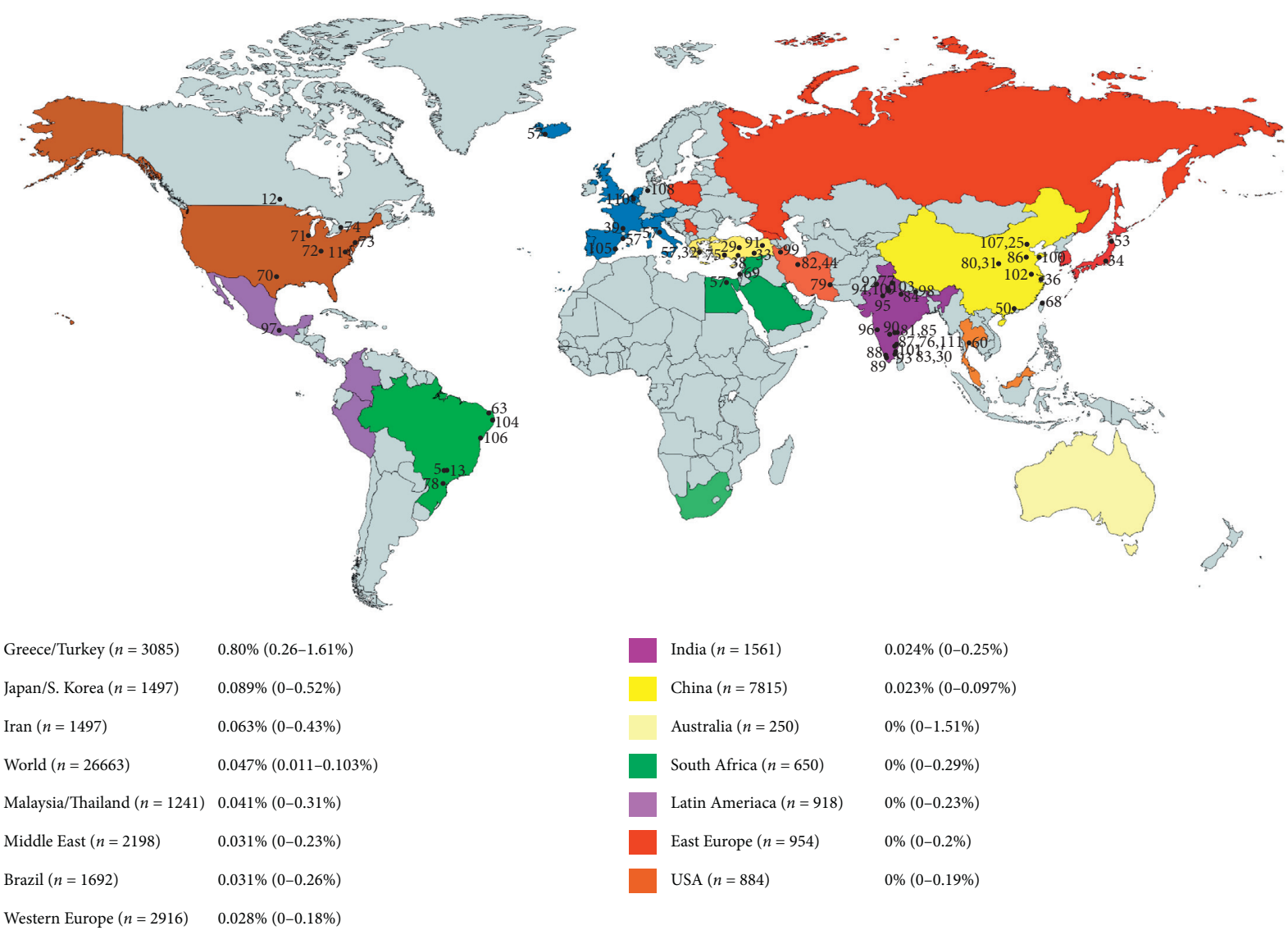

FIGURE 4: Global distribution of four-rooted maxillary first molars. All case reports and maxillary first molars with four roots identified in CBCT studies were marked on the world map. The regional differences in the occurrence rate were noted.

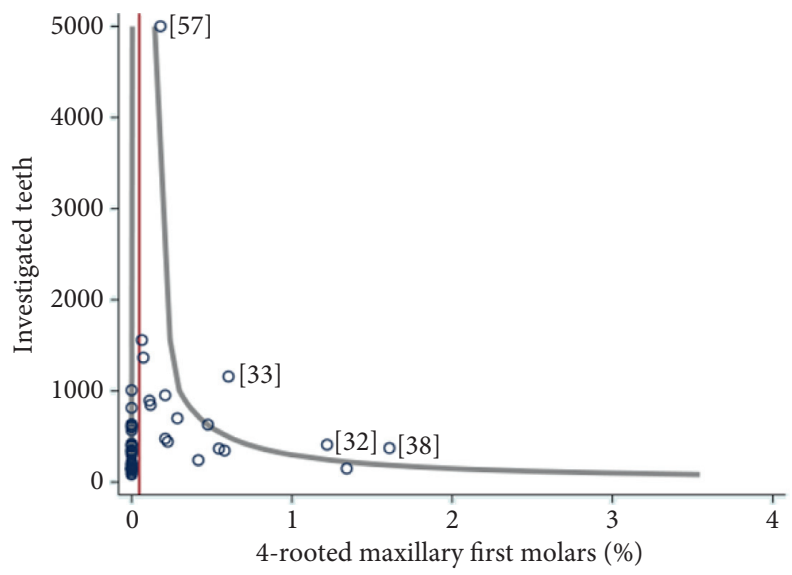

Figure 5: Funnel Plot. The vertical red line marks the median of all studies (0.047). The two gray curves mark the 95\% confidence interval, assuming a true percentage of 0.047 . The left gray line marks the lower limit of the $95 \% \mathrm{CI}$ and runs almost parallel to the $y$-axis; i.e., the lower boundary is 0 . Overall, the percentage of teeth with 4 roots is small $(0.047 \%)$; therefore, a large part of the studies shared $0 \%$.

continent (Figure 4). However, an anthropological conclusion reconstructing the human prehistoric colonization upon the teeth size as previously shown for C-shaped mandibular second molars [19] could not be demonstrated for four-rooted maxillary first molars.
However, the rarity of four-rooted maxillary first molars underlined by this review (in 26663 investigated maxillary first molars, only 47 exhibited four roots) caused the complete absence of studies concerning this topic [3]. Therefore, a systematic collection of case reports regarding 
TABLE 3: Evaluation of different anatomical characteristics, classification, and gender.

\begin{tabular}{|c|c|c|}
\hline \multirow{5}{*}{ Christie's Radicular Type $(n=46)[12,13]$} & Type I & $52.83 \%$ \\
\hline & Type II & $18.87 \%$ \\
\hline & Type III & $18.87 \%$ \\
\hline & Type IV & $1.88 \%$ \\
\hline & n.d. & $7.54 \%$ \\
\hline \multirow{4}{*}{ Pulpal floor type $(n=33)[14]$} & Type A & $45.45 \%$ \\
\hline & Type B & $51.52 \%$ \\
\hline & Type C & $0.00 \%$ \\
\hline & Type D & $3.03 \%$ \\
\hline \multirow{4}{*}{ Amount of root canals $(n=77)$} & $4 \mathrm{RCs}$ & $62.34 \%$ \\
\hline & $5 \mathrm{RCs}$ & $27.27 \%$ \\
\hline & $6 \mathrm{RCs}$ & $9.09 \%$ \\
\hline & $7 \mathrm{RCs}$ & $1.30 \%$ \\
\hline \multirow{2}{*}{ Tooth number $(n=54)$} & Left \#26 & $50.0 \%$ \\
\hline & Right \#16 & $50.0 \%$ \\
\hline \multirow{2}{*}{$\operatorname{Sex}(n=68)$} & Female & $39.71 \%$ \\
\hline & Male & $60.29 \% *$ \\
\hline
\end{tabular}

The information on the investigated case reports and the CBCT studies was combined. $* p<0.05$, n.d.:not defined, RC:root canal.

treatment protocols of maxillary molars with four roots was considered to be an appropriate study design. With this technique, the number of 53 teeth could be identified. This amount can be compared to the most extensive published studies on four-rooted maxillary second molars with either 22 [12] or 25 investigated teeth [14]. One of the further strengths of the present review is the combination of CBCT studies and case reports, which results in, e.g., 77 investigated teeth concerning the number of root canals. Thus, upon this data, an anatomical description of four-rooted maxillary molars should be possible.

The radicular structure of four-rooted maxillary first molars is commonly described with Christie's classification (Figure 1), although it was designed mainly upon maxillary second molars [12]. In the present review, 37.7\% of the case report's authors used Christie's characterization. But, even after the application of Christie's defined radiographic regulation, $7.54 \%$ of the teeth could not be classified upon the different types. These unclassified teeth had either fusion between roots [94] or three buccal (two mesiobuccal and one distobuccal) Radixes [88, 91]. Therefore, Christie's classification cannot be transferred from maxillary second molars with four roots to four-rooted maxillary first molars without neglecting some teeth.

Regarding fusions, this review agreed with Versiani et al. in 2012, who pointed out that Christie's configuration is not feasible because fusions might occur in different levels of all roots (Figure 1) [14]. On the other hand, Versiani's suggested classification also ignored the description of three buccal and one palatal root. But, Versiani investigated fourrooted maxillary second molars, and these teeth exhibit probably no third buccal radicular structure. The main question is whether a second mesiobuccal root could be classified as a mesiopalatal, even if both structures can be found in five-rooted maxillary teeth [113].

However, the definition of Carlsen and Alexandersen, which is rarely used in scientific publications concerning maxillary molars with four roots, included the possibility of three buccal roots but was designed in vitro and on untreated teeth $[15,16]$. But, their anatomical characterization described the radicular structures based on coronal anomalies [15], which might be decayed under clinical circumstances. In summary, all standard classifications were not entirely feasible or established for the description of four-rooted maxillary first molars. To cover all variants of four- or even five-rooted maxillary molars [113] or O-shaped teeth [114], new classifications have to be designed, which should also support the clinical and radiographic diagnostic.

In relation to Versiani's pulpal chamber floor classification, which defined the localization of the root canal orifices geometrically, the analyzed case reports documented the main allocation on Type A (irregular quadrilateral) and Type B (trapezoid) (Figure 2). With respect to the sample size of 33 teeth, this data corresponds to findings in fourrooted maxillary second molars where Types A and B were exhibited mainly [14]. However, the majority of maxillary first molars with four roots showed one root canal per root, which also correlated with findings in four-rooted maxillary second molars [14]. Five root canals were identified in $27.27 \%$ of the cases, but also six or seven canals were found. It must be taken into account that the present review depends mainly on clinical case reports and that in vivo CBCT or in vitro $\mu \mathrm{CT}$ studies would have higher reliability.

Interestingly, the present review identified a significant difference in the occurrence of four roots in maxillary first molars between males and females. Comparable data for four-rooted maxillary second molars have not been published due to the rarity of these teeth. Therefore, and under consideration of the sample size of 68 analyzed teeth, the presented data could indicate a morphological discrepancy between the genders, which should be further investigated by worldwide CBCT population studies as mentioned above. These future anatomical studies should also focus on coronal anomalies (like a pronounced cusp of Carabelli) [15] as well as on the occurrence of enamel pearls in four-rooted maxillary molars. Coronal factors were often mentioned to support the diagnostic of radicular alterations but have to be scientifically proven. In the present review, only $15.1 \%$ of the 
case reports $[11,69,82,87,96,100,102,108]$ documented further anatomical variations combined with the exhibition of four roots (Table 2). However, dental professionals should be aware of this rare anomaly to avoid treatment errors that result oftentimes in endodontic retreatments $[93,95,98,101,115]$.

\section{Conclusion}

The occurrence rate for 4-rooted maxillary first molars in humans is $0.047 \%$. By collecting population-based CBCT studies and case reports, this quadrangular anomaly was described with mainly four root canals. The root canal orifices on the pulpal chamber floor are located in trapezoid or irregular quadrilateral shape.

\section{Data Availability}

All underlying data can be found in the manuscript.

\section{Conflicts of Interest}

The authors declare no conflicts of interest related to this study.

\section{Acknowledgments}

The authors would like to thank Mrs. Monika Paidipamula for proofreading and Mrs. Anita Kurth for the graphical design of the figures.

\section{Supplementary Materials}

Supplementary Table 1:the predefined inclusion and exclusion criteria for the literature selection of populationbased CBCT studies and case reports concerning fourrooted maxillary first molars. Supplementary Table 2: the investigated CBCT studies in regional subgroups to evaluate the worldwide occurrence rate of maxillary first molars with four roots. The final results were illustrated in Figure 4. The analyzed regional subgroups are in alphabetical order Australia (Suppl. Table 2.10), Brazil (Suppl. Table 2.6), China (Suppl. Table 2.9), East Europe (Suppl. Table 2.13), Greece/ Turkey/Cyprus (Suppl. Table 2.1), India (Suppl. Table 2.8), Iran (Suppl. Table 2.3), Japan/South Korea (Suppl. Table 2.2), Latin America (Suppl. Table 2.12), Malaysia/Thailand (Suppl. Table 2.4), Middle East (Suppl. Table 2.5), Western Europe (Suppl. Table 2.7), South Africa (Suppl. Table 2.11), and USA (Suppl. Table 2.14). (Supplementary Materials)

\section{References}

[1] G. Carabelli, Systemisches Handbuch der Zahnheilkunde. Anatomie des Mundes, Vol. 2, Braunmuller und Seidel, Wien, Austria, 1844.

[2] F. S. Weine, Endodontic Therapy, The C.V. Mosby Company, St. Louis, USA, 1972.

[3] B. M. Cleghorn, W. H. Christie, and C. C. S. Dong, "Root and root canal morphology of the human permanent maxillary first molar:a literature review," Journal of Endodontics, vol. 32, no. 9, pp. 813-821, 2006.

[4] J. N. R. Martins, D. Marques, E. J. N. L. Silva, J. Carames, A. Mata, and M. A. Versiani, "Second mesiobuccal root canal in maxillary molars-A systematic review and meta-analysis of prevalence studies using cone beam computed tomography," Archives of Oral Biology, vol. 113, Article ID 104589, 2019.

[5] J. Barbizam, R. Ribeiro, and M. Filho, "Unusual anatomy of permanent maxillary molars," Journal of Endodontics, vol. 30, no. 9, pp. 668-671, 2004.

[6] S. Paksefat and S. Rahimi, "Root canal treatment of a tworooted C-shaped maxillary first molar:a case report," Iranian Endodontic Journal, vol. 9, no. 4, pp. 301-303, 2014.

[7] S. Jaikrishnan, J. Kottoor, J. Mathew, S. R. Kumar, S. George, and K. Hari, "Evaluation and endodontic management of a patient with 6 single-rooted molars:a case report," General Dentistry, vol. 63, no. 5, pp. 35-37, 2015.

[8] S. Holderrieth and C. R. Gernhardt, "Maxillary molars with morphologic variations of the palatal root canals:a report of four cases," Journal of Endodontics, vol. 35, no. 7, pp. 1060-1065, 2009.

[9] J. Kottoor, N. Velmurugan, and S. Surendran, "Endodontic management of a maxillary first molar with eight root canal systems evaluated using cone-beam computed tomography scanning:a case report," Journal of Endodontics, vol. 37, no. 5, pp. 715-719, 2011.

[10] G. P. Badole, M. M. Warhadpande, P. R. Shenoi, C. Lachure, and S. G. Badole, "A rare root canal configuration of bilateral maxillary first molar with 7 root canals diagnosed using cone-beam computed tomographic scanning:a case report," Journal of Endodontics, vol. 40, no. 2, pp. 296-301, 2014.

[11] M. E. Thews, W. B. Kemp, and C. R. Jones, "Aberrations in palatal root and root canal morphology of two maxillary first molars," Journal of Endodontics, vol. 5, no. 3, pp. 94-96, 1979.

[12] W. H. Christie, M. D. Peikoff, and H. M. Fogel, "Maxillary molars with two palatal roots:a retrospective clinical study," Journal of Endodontics, vol. 17, no. 2, pp. 80-84, 1991.

[13] F. Baratto-Filho, L. F. Fariniuk, E. L. Ferreira, J. D. Pecora, A. M. Cruz-Filho, and M. D. Sousa-Neto, "Clinical and macroscopic study of maxillary molars with two palatal roots," International Endodontic Journal, vol. 35, no. 9, pp. 796-801, 2002.

[14] M. A. Versiani, J. D. Pecora, and M. D. De Sousa-Neto, "Root and root canal morphology of four-rooted maxillary second molars:a micro-computed tomography study," Journal of Endodontics, vol. 38, no. 7, pp. 977-982, 2012.

[15] O. Carlsen and V. Alexandersen, "Radix mesiolingualis and radix distolingualis in a collection of permanent maxillary molars," Acta Odontologica Scandinavica, vol. 58, no. 5, pp. 229-236, 2000.

[16] O. Carlsen and V. Alexandersen, "Radix paramolaris and radix distomolaris in Danish permanent maxillary molars," Acta Odontologica Scandinavica, vol. 57, no. 5, pp. 283-289, 1999.

[17] D. Moher, A. Liberati, J. Tetzlaff, and D. G. Altman, "Preferred reporting items for systematic reviews and metaanalyses:the PRISMA statement," BMJ, vol. 339, no. jul21 1, p. 339:b2535, 2009.

[18] Z. Altman, S. Moola, K. Lisy, D. Riitano, and C. Tufanaru, "Methodological guidance for systematic reviews of observational epidemiological studies reporting prevalence and 
cumulative incidence data," International Journal of Evidence Based Healthcare, vol. 13, no. 3, pp. 147-153, 2015.

[19] J. N. R. Martins, D. Marques, E. J. N. L. Silva, J. Carames, A. Mata, and M. A. Versiani, "Prevalence of C-shaped canal morphology using cone beam computed tomography - a systematic review with meta-analysis," International Endodontic Journal, vol. 52, no. 11, pp. 1556-1572, 2019.

[20] Q.-H. Zheng, Y. Wang, X.-D. Zhou, Q. Wang, G.-N. Zheng, and D.-M. Huang, "A cone-beam computed tomography study of maxillary first permanent molar root and canal morphology in a Chinese population," Journal of Endodontics, vol. 36, no. 9, pp. 1480-1484, 2010.

[21] R. Zhang, H. Yang, X. Yu, H. Wang, T. Hu, and P. M. H. Dummer, "Use of CBCT to identify the morphology of maxillary permanent molar teeth in a Chinese subpopulation," International Endodontic Journal, vol. 44, no. 2, pp. 162-169, 2011.

[22] Y. Kim, S.-J. Lee, and J. Woo, "Morphology of maxillary first and second molars analyzed by cone-beam computed tomography in a Korean population:variations in the number of roots and canals and the incidence of fusion," Journal of Endodontics, vol. 38, no. 8, pp. 1063-1068, 2012.

[23] M. F. Abed, S. A. R. Kolahdouzan, and S. P. Hashemi, "Usage of cone-beam computed tomography (CBCT) to evaluate root and canal morphology of maxillary first molar," Bulletin of Environment, Pharmacology and Life Sciences, vol. 2, no. 2, pp. 19-23, 2013.

[24] G. Plotino, L. Tocci, N. M. Grande et al., "Symmetry of root and root canal morphology of maxillary and mandibular molars in a white population:a cone-beam computed tomography study in vivo," Journal of Endodontics, vol. 39, no. 12, pp. 1545-1548, 2013.

[25] Y. N. Jing, X. Ye, D. G. Liu, Z. Y. Zhang, and X. C. Ma, "Cone-beam computed tomography was used for study of root and canal morphology of maxillary first and second molars," Beijing Da Xue Xue Bao Yi Xue Ban, vol. 46, no. 9, pp. 958-962, 2014.

[26] E. J. N. L. Silva, Y. Nejaim, A. I. V. Silva, F. Haiter-Neto, A. A. Zaia, and N. Cohenca, "Evaluation of root canal configuration of maxillary molars in a Brazilian population using cone-beam computed tomographic imaging:an in vivo study," Journal of Endodontics, vol. 40, no. 2, pp. 173-176, 2014.

[27] J. Guo, A. Vahidnia, P. Sedghizadeh, and R. Enciso, "Evaluation of root and canal morphology of maxillary permanent first molars in a North American population by cone-beam computed tomography," Journal of Endodontics, vol. 40, no. 5, pp. 635-639, 2014.

[28] C. Estrela, M. R. Bueno, G. S. Couto et al., "Study of root canal anatomy in human permanent teeth in A subpopulation of Brazil's center region using cone-beam computed tomography - Part 1," Brazilian Dental Journal, vol. 26, no. 5, pp. 530-536, 2015.

[29] S. Demırbuga, S. K. Buyuk, A. E. Serkerc1 et al., "Bir grup türk populasyonunda üst birinci ve ikinci büyük azı dişlerinin kök kanal konfigürasyonlarının konik işınlı bilgisayarlı tomografi ile incelenmesi," Turkiye Klinikleri Journal of Dental Sciences, vol. 21, no. 1, pp. 1-9, 2015.

[30] G. Felsypremila, T. S. Vinothkumar, and D. Kandaswamy, "Anatomic symmetry of root and root canal morphology of posterior teeth in Indian subpopulation using cone beam computed tomography:a retrospective study," $E$ ropean Journal of Dentistry, vol. 09, no. 4, pp. 500-507, 2015.
[31] Y. Gu, W. Wang, and L. Ni, "Four-rooted permanent maxillary first and second molars in a northwestern Chinese population," Archives of Oral Biology, vol. 60, no. 6, pp. 811-817, 2015.

[32] G. E. Nikoloudaki, T. G. Kontogiannis, and N. P. Kerezoudis, "Evaluation of the root and canal morphology of maxillary permanent molars and the incidence of the second mesiobuccal root canal in Greek population using cone-beam computed tomography," The Open Dentistry Journal, vol. 9, no. Suppl 2:M3, pp. 267-272, 2015.

[33] M. Altunsoy, E. Ok, B. G. Nur, O. S. Aglarci, E. Gungor, and M. Colak, "Root canal morphology analysis of maxillary permanent first and second molars in a southeastern Turkish population using cone-beam computed tomography," Journal of Dental Sciences, vol. 10, no. 4, pp. 401-407, 2015.

[34] H. Nakazawa, T. Baba, and Y. Tsujimoto, "Analysis of root and root canal morphology of maxillary first and second molars using multi-detector CT in Japanese population," The Japanese Journal of Conservative Dentistry, vol. 58, no. 5, pp. 406-415, 2015.

[35] E. Beshkenadze and N. Chipashvili, "Anatomo-morphological features of the root canal system in Georgian population-cone-beam computed tomography study," Georgian Medical News, vol. 10, no. 247, pp. 7-14, 2015.

[36] X.-M. Tian, X.-W. Yang, L. Qian, B. Wei, and Y. Gong, "Analysis of the root and canal morphologies in maxillary first and second molars in a Chinese population using conebeam computed tomography," Journal of Endodontics, vol. 42, no. 5, pp. 696-701, 2016.

[37] M. Tanvi, N. Vimala, and M. Lalitagauri, "Evaluation of the root morphology of maxillary permanent first and second molars in an Indian subpopulation using cone-beam computed tomography," IOSR Journal of Dental and Medical Sciences, vol. 15, no. 10, pp. 51-56, 2016.

[38] A. Kalender, B. Celikten, P. Tufenkci et al., "Cone beam computed tomography evaluation of maxillary molar root canal morphology in a Turkish Cypriot population," Biotechnology and Biotechnological Equipment, vol. 30, no. 1, pp. 145-150, 2016.

[39] P. Monsarrat, B. Arcaute, O. A. Peters et al., "Interrelationships in the variability of root canal anatomy among the permanent teeth:a full-mouth approach by cone-beam ct," PLoS One, vol. 11, no. 10, Article ID e0165329, 2016.

[40] M. Naseri, Y. Safi, A. Akbarzadeh Baghban, A. Khayat, and L. Eftekhar, "Survey of anatomy and root canal morphology of maxillary first molars regarding age and gender in an Iranian population using cone-beam computed tomography," Iranian Endodontic Journal, vol. 11, no. 4, pp. 298-303, 2016.

[41] H. X. Liu and Y. Z. Ma, "Cone beam computed tomography study of the root canal morphology of maxillary first molars," Journal of Prevention and Treatment for Stomatological Diseases, vol. 24, no. 8, pp. 498-500, 2016.

[42] A. A. Irhaim, "Evaluation of the root and canal morphology of permanent maxillary first molars using cone beam computed tomography in a sample of patients treated at the wits oral health centre," Master Thesis, University of the Witwatersrand, Johannesburg, South Africa, 2016.

[43] R. P. Mohan, M. S. Thomas, N. Shetty, J. Ahmed, G. Pallippurath, and A. Tallada, "Evaluation of the root and canal morphology of maxillary first and second molar using cone beam computed tomography:a retrospective study," World Journal of Dentistry, vol. 8, no. 2, pp. 134-138, 2017. 
[44] Z. Ghoncheh, B. M. Zade, and M. J. Kharazifard, "Root morphology of the maxillary first and second molars in an Iranian population using cone beam computed tomography," Journal of Dentistry (Tehran, Iran), Tehran University of Medical Sciences, vol. 14, no. 3, pp. 115-122, 2017.

[45] A. Khademi, A. Zamani Naser, Z. Bahreinian, M. Mehdizadeh, M. Najarian, and S. Khazaei, "Root morphology and canal configuration of first and second maxillary molars in a selected Iranian population:a cone-beam computed tomography evaluation," Iranian Endodontic Journal, vol. 12, no. 3, pp. 288-292, 2017.

[46] Y.-H. Lin, H.-N. Lin, C.-C. Chen, and M.-S. Chen, "Evaluation of the root and canal systems of maxillary molars in Taiwanese patients:a cone beam computed tomography study," Biomedical Journal, vol. 40, no. 4, pp. 232-238, 2017.

[47] K. Olczak and H. Pawlicka, "The morphology of maxillary first and second molars analyzed by cone-beam computed tomography in a polish population," BMC Medical, vol. 17, no. 1, 68 pages, 2017.

[48] M. Perez-Heredia, C. M. Ferrer-Luque, M. Bravo, P. CasteloBaz, M. Ruíz-Piñón, and P. Baca, "Cone-beam computed tomographic study of root anatomy and canal configuration of molars in a Spanish population," Journal of Endodontics, vol. 43, no. 9, pp. 1511-1516, 2017.

[49] A. M. Ghobashy, M. M. Nagy, and A. A. Bayoumi, "Evaluation of root and canal morphology of maxillary permanent molars in an Egyptian population by cone-beam computed tomography," Journal of Endodontics, vol. 43, no. 7, pp. 1089-1092, 2017.

[50] H. Wang, B. W. Ci, H. Y. Yu et al., "Evaluation of root and canal morphology of maxillary molars in a Southern Chinese subpopulation:a cone-beam computed tomographic study," International Journal of Clinical and Experimental Medicine, vol. 10, no. 4, pp. 7030-7039, 2017.

[51] S. Al-Shehri, S. Al-Nazhan, S. Shoukry, E. Al-Shwaimi, R. AlSadhan, and B. Al-Shemmery, "Root and canal configuration of the maxillary first molar in a Saudi subpopulation:a conebeam computed tomography study," Saudi Endodontic Journal, vol. 7, no. 2, pp. 69-76, 2017.

[52] A. H. Al-Kadhim, Z. A. Rajionb, N. A. B. Malika, and A. B. Jaafara, "Morphology of maxillary first molars analyzed by cone-beam computed tomography among Malaysian: variations in the number of roots and canals and the incidence of fusion," International Medical Journal Malaysia, vol. 16, no. 2, pp. 33-39, 2017.

[53] A. Ogawa and S. Seki, "Evaluation of root anatomy and root canal configurations of mandibular second molars in a Japanese population using dental cone-beam computed tomography," The Journal of Japan Endodontic Association, vol. 38, no. 1, pp. 57-62, 2017.

[54] Y. Zhang, H. Xu, D. Wang et al., "Assessment of the second mesiobuccal root canal in maxillary first molars:a cone-beam computed tomographic study," Journal of Endodontics, vol. 43, no. 12, pp. 1990-1996, 2017.

[55] J. N. R. Martins, D. Marques, H. Francisco, and J. Carames, "Gender influence on the number of roots and root canal system configuration in human permanent teeth of a Portuguese subpopulation," Quintessence International, vol. 49, no. 2, pp. 103-111, 2018.

[56] S. Razumova, A. Brago, L. Khaskhanova, A. Howijieh, H. Barakat, and A. Manvelyan, "A cone-beam computed tomography scanning of the root canal system of permanent teeth among the moscow population," International Journal of Dentistry, vol. 2018, Article ID 2615746, 6 pages, 2018.
[57] J. N. R. Martins, M.-B. A. M. Alkhawas, Z. Altaki et al., "Worldwide analyses of maxillary first molar second mesiobuccal prevalence:a multicenter cone-beam computed tomographic study," Journal of Endodontics, vol. 44, no. 11, pp. 1641-1649, 2018.

[58] S. A. B. Salem, S. M. Ibrahim, and A. M. Abdalsamad, "Prevalence of second mesio-buccal canal in maxillary first and second molars in Egyptian population using CBCT (A cross-sectional study)," Acta Scientific Dental Science, vol. 2, no. 9, pp. 64-68, 2018.

[59] A. C. Arbildo Villalta, "Evaluacíon de la morfologia radicular y del Sistema de conductos en la primera molar superior permanente en el hospital Luiz Nazario Sáenz, Periodo 20142018," Ph. D Thesis, Facultad de medicina humana y ciencia de la salud, Escuela professional de estomatologia, Lima, Peru, 2018.

[60] R. Ratanajirasut, A. Panichuttra, and S. Panmekiate, "A cone-beam computed tomographic study of root and canal morphology of maxillary first and second permanent molars in a Thai population," Journal of Endodontics, vol. 44, no. 1, pp. 56-61, 2018.

[61] E. Salzmann, 3-D analyse der wurzelanatomie und kanalmorphologie des ersten oberkiefer molaren anhand von cbct daten der universitätszahnklinik wien, Ph.D Thesis, Medizinische Universität Wien, Wien, Austria, 2018.

[62] J. Y. Y. Pan, A. Parolia, S. R. Chuah, S. Bhatia, S. Mutalik, and A. Pau, "Root canal morphology of permanent teeth in a Malaysian subpopulation using cone-beam computed tomography," BMC Oral Health, vol. 19, no. 1, p. 14, 2019.

[63] G. T. M. Candeiro, S. D. S. Gonçalves, L. L. A. Lopes et al., "Internal configuration of maxillary molars in a subpopulation of Brazil's Northeast region:a CBCT analysis," Brazilian Oral Research, vol. 33, no. 1, Article ID e082, 2019.

[64] N. T. Mohara, M. S. Coelho, N. V. De Queiroz et al., "Root anatomy and canal configuration of maxillary molars in a Brazilian subpopulation:a $125-\mu \mathrm{m}$ cone-beam computed tomographic study," European Journal of Dentistry, vol. 13, no. 1, pp. 82-87, 2019.

[65] R. Kewalramani, C. S. Murthy, and R. Gupta, "The second mesiobuccal canal in three-rooted maxillary first molar of Karnataka Indian sub-populations:a cone-beam computed tomography studyfirst molar of Karnataka Indian subpopulations:a cone-beam computed tomography study," Journal of Oral Biology and Craniofacial Research, vol. 9, no. 4, pp. 347-351, 2019.

[66] M. Popovic, S. Zivanovic, T. Vucicevic, M. Grujovic, M. Papic, and T. Kanjevac, "Cone-beam computed tomography study of tooth root and canal morphology of permanent molars in a Serbian population," Vojnosanitetski Pregled, vol. 1, no. 00, p. 100, 2019.

[67] M. Mashyakhy and G. Gambarini, "Root and root canal morphology differences between genders:a comprehensive in-vivo CBCT study in a Saudi population," Acta Stomatologica Croatica, vol. 53, no. 3, pp. 231-246, 2019.

[68] L. T. Tzeng, M. C. Chang, S. H. Chang, C. C. Huang, Y. J. Chen, and J. H. Jeng, "Analysis of root canal system of maxillary first and second molars and their correlations by cone beam computed tomography," Journal of the Formosan Medical Association, vol. S0929-6646, no. 19, pp. 3076230764, 2019.

[69] A. Stabholz and S. Friedman, "Endodontic therapy of an unusual maxillary permanent first molar," Journal of Endodontics, vol. 9, no. 7, pp. 293-295, 1983. 
[70] M. Wong, "Maxillary first molar with three palatal canals," Journal of Endodontics, vol. 17, no. 6, pp. 298-299, 1991.

[71] E. L. Jacobsen and C. Nii, "Unusual palatal root canal morphology in maxillary molars," Dental Traumatology, vol. 10, no. 1, pp. 19-22, 1994.

[72] P. M. Di Fiore, "A four-rooted quadrangular maxillary molar," Journal of Endodontics, vol. 25, no. 10, pp. 695-697, 1999.

[73] F. Maggiore, Y. T. Jou, and S. Kim, "A six-canal maxillary first molar:case report," International Endodontic Journal, vol. 35, no. 5, pp. 486-491, 2002.

[74] Y. Nahimas and P. Bery, "Double palatal roots," Ontario Dentist, no. Oct, pp. 24-26, 2006.

[75] N. Adanir, "An unusual maxillary first molar with four roots and six canals:a case report," Australian Dental Journal, vol. 52, no. 4, pp. 333-335, 2007.

[76] P. Ravishanker and K. Prashanthi, "Unusual Maxillary First Molar with two palatal roots:a case report," The Internet Journal of Dental Science, vol. 7, no. 1, pp. 1-4, 2008.

[77] A. Gandhi and N. Madan, "Aberration in root canal morphology of maxillary first molar- A case report," The Internet Journal of Dental Science, vol. 9, no. 1, pp. 1-4, 2009.

[78] F. S. F. Tomazinho, F. Baratto-Filho, S. Zaitter, D. P. Leonardi, and C. C. Gonzaga, "Unusual anatomy of a maxillary first molar with two palatal roots:a case report," Journal of Oral Science, vol. 52, no. 1, pp. 149-153, 2010.

[79] M. Salapoor and N. Farhad Mollashahi, "Maxillary first molar with two separate palatal roots:a case report," Journal of Dental Medicine, vol. 23, no. 4, pp. 198-201, 2010.

[80] W. He, K. Wei, J. Chen, and Q. Yu, "Endodontic treatment of maxillary first molars presenting with unusual asymmetric palatal root morphology using spiral computerized tomography:a case report," Oral Surgery, Oral Medicine, Oral Pathology, Oral Radiology, and Endodontology, vol. 109, no. 1, pp. e55-e59, 2010.

[81] R. V. S. Chakradhar Raju, V. Chandrasekhar, C. Singh, and S. Pasari, "Maxillary molar with two palatal roots:two case reports," Journal of Conservative Dentistry, vol. 13, no. 1, pp. 58-61, 2010.

[82] H. Moghaddas and Z. Alizadeh Tabari, "Palatal cervical enamel projection in a four rooted maxillary first molar:a case report," Research Journal of Biological Sciences, vol. 5, no. 7, pp. 508-511, 2010.

[83] J. Kottoor, N. Velmurugan, S. Ballal, and A. Roy, "Fourrooted maxillary first molar having $\mathrm{C}$-shaped palatal root canal morphology evaluated using cone-beam computerized tomography:a case report," Oral Surgery, Oral Medicine, Oral Pathology, Oral Radiology, and Endodontology, vol. 111, no. 5, pp. e41-e45, 2011.

[84] A. Singh, P. Singh, and K. Loomba, "Unusual anatomical variation in maxillary first molar and its management," Asian Journal of Oral Health and Allied Sciences, vol. 1, no. 2, pp. 134-137, 2011.

[85] S. J. Reddy, P. V. Ravichandra, P. Rajani, and L. Santoshi, "Palatal Possibilties:variations of palatal root canal configuration in maxillary molars - a Report of Two Cases," International Journal of Contemporary Dentistry, vol. 2, no. 2, pp. 85-90, 2011.

[86] Y. Du, I. Soo, and C. F. Zhang, "A case report of six canals in a maxillary first molar," Chinese Journal of Dental Research, vol. 14, no. 2, pp. 151-153, 2011.

[87] K. Madhuram, C. Dhanavel, V. Naveen, and R. Anbu, "Corono radicular anomaly in a maxillary first molar-a rare case report," Journal of Integrated Dentistry, vol. 1, no. 1, pp. 41-44, 2012.

[88] J. Kottoor, S. Nandini, and N. Velmurugan, "Maxillary first molar with three buccal roots evaluated with cone-beam computed tomography:a rare case report," General Dentistry, vol. 60, no. 6, pp. 404-407, 2012.

[89] J. Mathew, A. Devadathan, J. Jacob, G. Syriac, and J. C. Augustine, "Root canal treatment of a maxillary first molar with an unusual presentation of two palatal roots," Health Sciences, vol. 2, no. 3, JS006B pages, 2013.

[90] S. Rajalbandi, S. N. Shingte, K. J. Sundaresh, and R. Mallikarjuna, "Aberration in the palatal root of the maxillary first molar," BMJ Case Reports, vol. 2013, Article ID bcr-2013008641, 2013.

[91] K. Yesildal Yeter, M. S. Evcil, and L. B. Ayranci, "Maxillary first molar with aberrant morphology:four roots and six canals:case report," Turkiye Klinikleri Journal of Dental Sciences, vol. 19, no. 3, pp. 20-24, 2013.

[92] R. Aggarwal, Y. Kumar, R. Aggarwal, and H. Kaur, "Unusual anatomy of maxillary first molar with two palatal roots - confirmed with computed tomography:a case report," Indian Journal of Restorative Dentistry, vol. 2, no. 2, pp. 91-94, 2013.

[93] M. F. Ghani, A. V. R. Ebenezar, A. Narayanan, L. Martina, B. Mony, and T. Mageshwaran, "Root canal treatment of bilaterally symmetric four-rooted maxillary first molars with two palatal roots using cone beam computed tomography:a case report," ENDO (Lond Engl), vol. 7, no. 2, pp. 135-140, 2013.

[94] R. Sharma, K. Maroli, N. Sinha, and B. Singh, "An unusual maxillary molar with four roots and four buccal canals confirmed with the aid of spiral computed tomography:a case report," Journal of International Oral Health, vol. 6, no. 4, pp. 80-84, 2014.

[95] N. Kararia, V. Kararia, and M. Mathur, "Endodontic retreatment of a maxillary first molar with two palatal roots and three palatal canals," Journal of Advanced Medical and Dental Sciences Research, vol. 2, no. 2, pp. 180-183, 2014.

[96] D. Y. Shah and G. R. Jadhav, "Endodontic management of a maxillary molar with formation supradentalis:a case report," Journal of Conservative Dentistry :JCD, vol. 17, no. 5, pp. 481-482, 2014.

[97] C. D. Sanchez-Salas Orlando Isai, E. E. Jiminez-Valdes Brissa Itzel, and E. Gomez-Pizano, "Primer molar superior con dos raíces palatinas:reporte de un caso," Revista Endodoncia Actual, vol. 9, no. 1, pp. 16-20, 2014.

[98] R. Shrestha, "Permanent maxillary molar with two palatal roots:two case reports," Journal of Nepal Dental Association, vol. 15, no. 2, pp. 46-49, 2015.

[99] V. Asghari, S. Rahimi, N. Ghasemi, B. Talebzadeh, and A. Norlouoni, "Treatment of a maxillary first molar with two palatal roots," Iranian Endodontic Journal, vol. 10, no. 4, pp. 287-289, 2015.

[100] D. Wu and $\mathrm{H}$. Wu, "Maxillary first molar with twin-root canal in palatal side:two case reports," Hua Xi Kou Qiang Yi Xue Za Zhi, vol. 33, no. 3, pp. 329-330, 2015.

[101] V. L. Deepa, I. P. Bollu, B. Dhamaraju, and P. Yadla, "Detection and management of a complex canal configuration in mesiobuccal root of maxillary first molar using three dimensional imaging," Journal of International Society of Preventive and Community Dentistry, vol. 6, no. Suppl 1, pp. 75-78, 2016.

[102] N. Tao, Y. Mei, H. Zhou, and Y. Yang, "Treatment of a young permanent maxillary first molar with two palatal roots," JBR 
Journal of Clinical Diagnosis and Research, vol. 4, no. 1, Article ID 000128, 2016.

[103] G. Nayak, H. Aeran, and I. Singh, "Radix mesiolingualis and radix distolingualis:a case report of a tooth with an unual morphology," Restorative Dentistry and Endodontics, vol. 41, no. 4, pp. 322-331, 2016.

[104] L. D. N. P. Barreto and C. C. D. S. A. Lins, "Maxillary molar with two palatal canals type I:case report," MOJ Anatomy and Physiology, vol. 2, no. 3, pp. 51-53, 2016.

[105] I. Vazquez Natividad and V. J. Faus Llacer, "Tratamiento de conductos en un primer molar maxilar de cuarto raíces y cinco conductos," Revista SCO, vol. 6, pp. 36-40, 2016.

[106] E. Rodrigues, A. H. Braitt, B. F. Galvao, and E. J. N. L. Da Silva, "Maxillary first molar with 7 root canals diagnosed using cone-beam computed tomography," Restorative Dentistry and Endodontics, vol. 42, no. 1, pp. 60-64, 2017.

[107] C. Cao, C. Feng, Y. Wang, J. Xuan, and J. Yao, “A maxillary first molar with two palatal roots and an accessory canal diagnosed by CBCT," Journal of Practical Stomatology, vol. 33, no. 1, pp. 126-128, 2017.

[108] G. Magnucki and W. A. Kahl, "Four rooted maxillary first molar with five root canals and two enamel pearls," The International Journal of Dental and Health Sciences, vol. 5, no. 4, pp. 600-605, 2018.

[109] B. Meena and M. Hasija, "A case report on non surgical management of rarely found maxillary first molar with four roots," International Journal of Oral Health Dentistry, vol. 4, no. 2, pp. 111-113, 2018.

[110] A. Schryvers, D. Govaerts, C. Politis, and P. Lambrechts, "Endodontic management of a maxillary first molar with two palatal roots:a case report," Australian Endodontic Journal, vol. 45, no. 3, pp. 420-425, 2018.

[111] A. Sriganesh and G. Saravana Priyan, "Endodontic management of maxillary first molar with an anatomical variation of two palatal canals:a case report," Indian Journal of Dental Research, vol. 30, no. 3, pp. 478-480, 2019.

[112] A. Kato, A. Ziegler, N. Higuchi, K. Nakata, H. Nakamura, and N. Ohno, "Aetiology, incidence and morphology of the C-shaped root canal system and its impact on clinical endodontics," International Endodontic Journal, vol. 47, no. 11, pp. 1012-1033, 2014.

[113] A. Borghesi, S. Michelini, E. Tononcelli, and R. Maroldi, "Five-rooted permanent maxillary second molar:CBCT findings of an extremely rare anatomical variant," Radiology Case Reports, vol. 14, no. 9, pp. 1113-1116, 2019.

[114] Y. Shin, Y. Kim, and B. D. Roh, "Maxillary first molar with an O-shaped root morphology:report of a case," International Journal of Oral Science, vol. 5, no. 4, pp. 22-24, 2013.

[115] G. Magnucki, "Endodontic retreatment of maxillary second molar with four roots," Case Reports in Dentistry, vol. 2019, Article ID 5348048, 5 pages, 2019. 